\title{
Changing antibiotic prescribing in General Practice - The results of the SIMPle process evaluation
}

\author{
Sinead Duane ${ }^{1}$, Christine Domegan ${ }^{2}$, Aoife Callan ${ }^{1,2}$, Sandra Galvin ${ }^{1}$, Martin Cormican ${ }^{3,4}$, Andrew W. Murphy ${ }^{1}$, Akke \\ Vellinga*1,4 \\ ${ }^{1}$ Discipline of General Practice, School of Medicine, NUI Galway, Ireland \\ 2 J.E. Cairnes School of Business and Economics, NUI Galway, Ireland \\ ${ }^{3}$ Department of Medical Microbiology, University Hospital Galway, Galway, Ireland \\ ${ }^{4}$ Discipline of Bacteriology, School of Medicine, NUI Galway, Ireland
}

Received: November 1, 2016

DOI: $10.5430 /$ ijh.v3n1p68
Accepted: February 4, $2017 \quad$ Online Published: February 28, 2017

URL: https://doi.org/10.5430/ijh.v3n1p68

\begin{abstract}
Objective: The Supporting the Improvement and Management of Prescribing for Urinary Tract Infections (SIMPle) study, a cluster randomized trial, sought to improve the quality of antibiotic prescribing for Urinary Tract Infections in General Practices located in the west of Ireland. This paper presents the process evaluation and intends to provide insights into the antibiotic prescribing behaviour of the General Practitioners (GPs) during the intervention. The paper discusses how the social marketing intervention developed as part of SIMPle resulted in GPs prescribing more first line antibiotics, the aim of the trial. However it also offers insights into why antibiotic prescriptions increased during the intervention period.

Methods: Fifteen GP interviews and summative intervention components including monitoring practice participation and information provided to the study team. Thematic analysis was used to analyze transcripts.

Results: GPs recognized the value of consultation coding to produce practice specific audit and feedback reports including current antibiotic prescribing and providing evidence which motivated change.

Conclusions: The SIMPle study was successfully integrated into routine care. By simplifying GPs' access to up to date evidence on their prescribing behaviors, GPs can be positively influenced beyond the context of an intervention.
\end{abstract}

Key Words: Antibiotics, General Practice, Prescribing, Process evaluation

\section{INTRODUCTION}

Antibiotic resistance (ABR) is a major public health challenge, the scale of which warranted the adoption of a global action plan by the 2015 World Health Assembly. ${ }^{[1]}$ ABR presents society with a "collective action problem" whereby the more we use antibiotics the greater the evolutionary pressure placed on bacteria. ${ }^{[2]}$ The over prescription and inappropriate use of antibiotics in human health care are key contributors. ${ }^{[3]}$

Society's overreliance on antibiotics has resulted in longer duration of illnesses, treatment failure and higher cost of health care for patients. These negative consequences have also placed an unnecessary burden on health care systems across the globe through reconsultations, additional and prolonged hospital stays and the need to provide alternative

*Correspondence: Akke Vellinga; Email: Akke.vellinga@nuigalway.ie; Address: NUI Galway, 1 Distillery Road, Galway, Ireland. 
treatments for what were in some cases relatively simple infections. It is difficult to place an economic cost on ABR worldwide, however, it is estimated that antibiotic resistant infections cost the United States health system alone an estimated $21-34$ billion annually. ${ }^{[4]}$ To reduce the financial and administrative burden on health systems across the globe action must be taken to address this issue.

A large amount of antibiotic prescribing takes place in General Practice in the community and mobilizing sustainable change at this level could have a knock on effect on other layers in the health system. For instance, General Practitioners (GPs) are a key stakeholder in the fight against ABR. In Ireland, $80 \%$ of antibiotic prescribing takes place in the community by GPs. ${ }^{[5]}$ GPs, as health professionals, act as role models on how to use antibiotics and patients learn about antibiotics from the prescriptions they receive. ${ }^{[6]}$ Therefore, GPs have the power to drive forward the change process.

Behavioral interventions are needed to reduce the risk of ABR in the future. ${ }^{[7]}$ High quality evaluations of these behavioral interventions are also essential to quantify their impact. Randomized Controlled Trials (RCTs) are the most rigorous intervention evaluation method. ${ }^{[8]}$ RCTs focus on primary and secondary outcomes but may not capture the effects of the interaction between elements; the process or processes that effect change or explain why an intervention is successful or not in a real life setting. ${ }^{[9,10]}$ The leading Cochrane systematic review examining interventions to improve antibiotics prescribing practices recommends for further research to be conducted on which elements within interventions are most effective. ${ }^{[11]}$ Knowledge of the elements impacting most on behaviors can assist in the interpretation of outcome results and make recommendations on how interventions can be integrated into practice. Process evaluations are an essential method of assessing these impacts. They can explore the causal mechanisms effecting the change being measured and therefore help our understanding of why complex interventions are successful before they are scaled up. ${ }^{[12]}$ This paper presents the findings from the process evaluation which was embedded within the SIMPle study (Supporting the Improvement and Management of Prescribing for Urinary Tract Infections). The SIMPle study was designed using the principles of social marketing and informed by qualitative formative research. ${ }^{[13]}$ The SIMPle study aimed to improve antibiotics prescribing for Urinary Tract Infections (UTI) in primary care through a multifaceted complex intervention which consisted of interactive workshops facilitated in 30 General Practices in the west of Ireland. During these workshops, participating GPs were requested to code their UTI consultations on their patient management software and were given the opportunity to discuss their antibiotic pre-

Published by Sciedu Press scribing behaviors compared to other participating practices. Participants were then encouraged to prescribe nitrofurantoin as first line treatment for UTI. By coding UTI consultations practices were provided with practice specific audit and feedback reports on their antibiotic prescribing behaviors, specifically what antibiotics they were prescribing for UTI compared to others and resistance levels in their area. All recommendations discussed within the workshops could be integrated into routine practice, ensuring the burden on practices was minimal.

Consultation coding also allowed for seamless electronic transfer of data between General Practices and the researchers. ${ }^{[14,15]}$ The study's CONSORT diagram is provided in Figure 1. SIMPle's primary outcome was to increase the number of first-line antibiotic prescriptions, as recommended in the Guidelines for Antimicrobial Prescribing in Primary Care in Ireland (2011), for suspected UTIs in primary care by $10 \%$ in adult patients. ${ }^{[14]}$ The SIMPle study achieved an increase in first line prescriptions for UTI consultation by 20 absolute percentage points. ${ }^{[15]}$ The SIMPle study illustrates the positive impact complex interventions integrated into routine care can have on antibiotics prescribing behaviour. ${ }^{[15]}$ However, upon further analysis the results of the SIMPle study indicate two unintended outcomes. There was an increase in overall antibiotic prescriptions during the intervention period and GPs were less likely to switch from non-first line antibiotic treatments to the recommended nitrofurantoin than from trimethoprim (a first line treatment with high community resistance) to nitrofurantoin. Gaining some insight as to why these unintended outcomes happened may be quite complex ${ }^{[16]}$ but could be used to improve complex interventions in General Practice and make recommendations for practice in the future.

This process evaluation provides insights into the observed effects of introducing interactive workshops and audit and feedback reports to improve the quality of antibiotic prescribing for UTI. It will also help to specify the circumstances in which these elements are likely to be successful.

\section{METHODS}

This process evaluation followed Medical Research Council Guidelines ${ }^{[16]}$ and Saunders et al. (2005) six step iterative process evaluation method. ${ }^{[17]}$ It adopted a dual lens approach by including both formative (GP interviews) and summative (judgements about how the intervention was implemented) components. GP interviews were undertaken prior to the completion of analysis of the primary outcome, therefore the researchers did not know the outcome of the SIMPle study at the time of data collection. 


\subsection{Participants and procedures}

\subsubsection{GP participants}

Fifteen post intervention face to face interviews were conducted with GPs who participated in the SIMPle study. This process evaluation focused on intervention practices only ( $\mathrm{n}$ $=20)$, control practices $(n=10)$ were excluded. Participants were selected using a non-probability sampling strategy by observing their practices antibiotic prescribing behaviors. The SIMPle study measured change at the practice level (cluster trial design) and not the individual GP level. Prac- tices were ranked based on the percentage change in the proportion of nitrofurantoin prescribed between November 2013 and February 2014. Practices were categorized as those experiencing substantial improvement in the quality of their antibiotic prescribing (>15\%), some improvement (5\%-15\%) and a little/none at all (4\% or less). Study arm, size (number of full time GPs) and location (urban vs. rural) were also included as selection criteria. Table 1 summarizes the characteristics of the chosen practices.

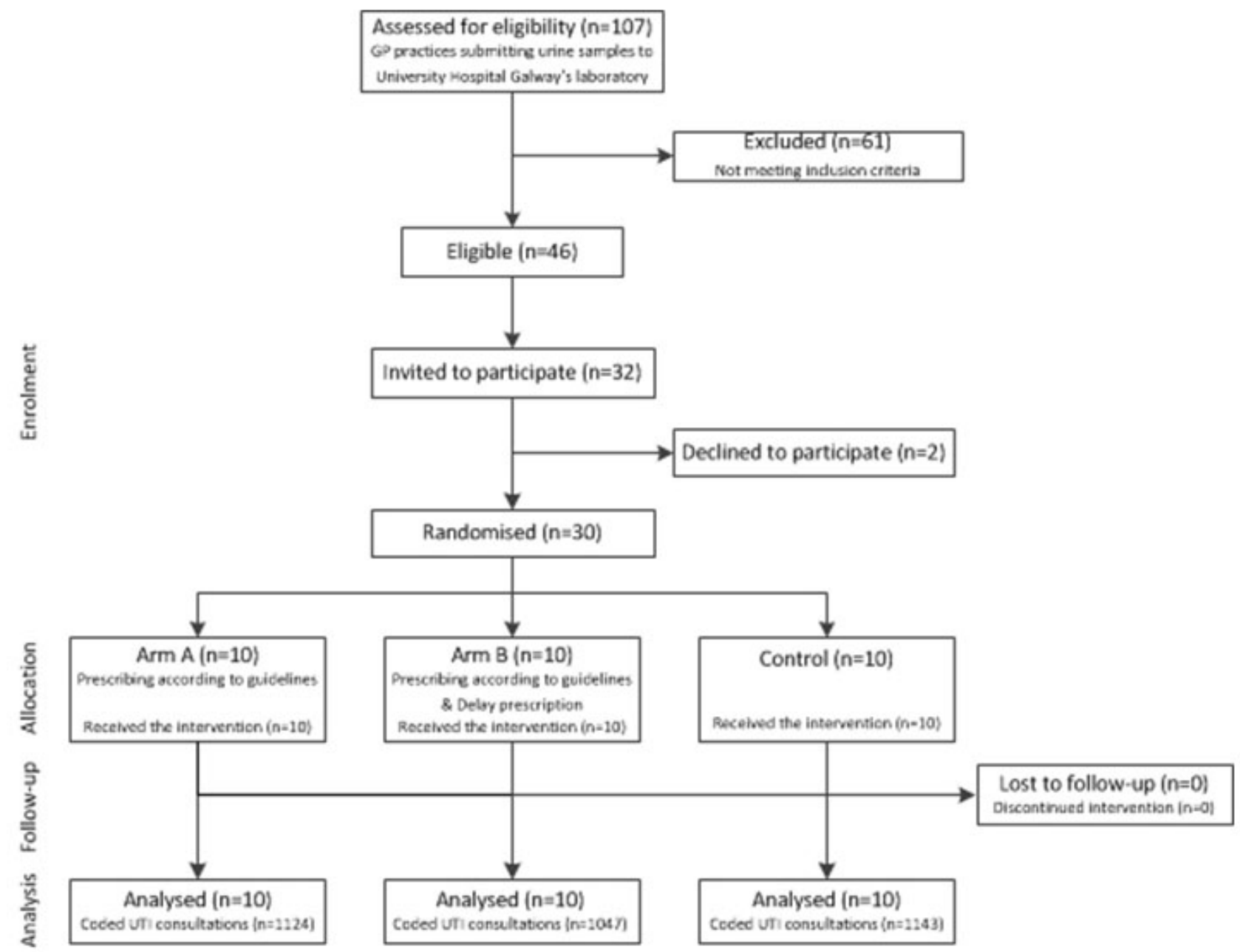

Figure 1. Consort flow diagram of the SIMPle study

\subsubsection{Interview procedures}

Fifteen GPs were interviewed across nine practices. Data collection continued until theoretical saturation was achieved. The same researcher facilitated all interviews, written consent was obtained from all participants and interviews were digitally recorded. All interviews were conducted within 3 weeks of the SIMPle study completion date.

\subsection{Analysis}

All interviews were transcribed verbatim. The analysis followed the principles of thematic analysis. The lead author (SD) conducted the preliminary analysis, and the other authors participated in the final analysis. All analysis was completed using NVIVO version 10 . The analysis process was informed by the Trans theoretical Model which uses two dimensions to understand the dynamics of change and how 
it occurs. ${ }^{[18]}$ First, Stages of Change examines aspects such as temporal and motivational issues. Second, Processes of Change identifies what events contribute to that change.

\subsection{Summative data collection}

Summative components included monitoring practice participation through appointment logs, team meetings relating to the progress of interactive workshops and analyzing the material provided during the workshops. The appointment logs included information on the number of attempts made via telephone to set up practice meetings. Additional observations included observing ease of scheduling appointments, attendance, and interaction during workshops and UTI coding through practices monthly audit and feedback reports.

Both GP interviews and summative components analysis focused on assessing fidelity (quality), dose delivered (completeness), dose reached (exposure), dose received (satisfaction) and reach (participation rate) of the SIMPle study.

Table 1. Overview of practices selected for interview

\begin{tabular}{lllll}
\hline $\begin{array}{l}\text { Intervention } \\
\text { Arm }\end{array}$ & $\begin{array}{l}\text { Location of } \\
\text { practice }\end{array}$ & $\begin{array}{l}\text { Percentage change in } \\
\text { Nitro prescribing }\end{array}$ & $\begin{array}{l}\text { No. of GPs within each practice } \\
\text { (FT = Full time, PT = Part time) }\end{array}$ & $\begin{array}{l}\text { No. of GPs who } \\
\text { participated in interview }\end{array}$ \\
\hline A & City & 40 & $2 \mathrm{FT} \mathrm{1PT}$ & 2 \\
A & Town & 29 & $3 \mathrm{FT}$ & 2 \\
A & City & 17 & $1 \mathrm{FT}$ & 1 \\
A & Town & 7 & $4 \mathrm{FT} \mathrm{3PT}$ & 2 \\
A & Town & 3 & $3 \mathrm{FT}$ & 2 \\
B & Rural & 41 & $1 \mathrm{FT}$ & 1 \\
B & City & 41 & $1 \mathrm{FT}$ & 1 \\
B & Town & 21 & $5 \mathrm{FT}$ & 2 \\
B & Rural & -6 & $3 \mathrm{FT}$ & 2 \\
Total & & & 15 & \\
\hline
\end{tabular}

Note. Nitro - Nitrofurentoin

\section{Results}

Combining the results of the formative and summative components of this process evaluation, several insights were made as to why GPs successfully improved the quality of antibiotic prescribing. These insights related to training and coding of consultations, the role of audit and feedback reports and the straightforward design of the SIMPle study.

\subsection{Training and coding of UTI consultations}

Consultation coding for an acute illness such as UTI was generally a new behaviour adopted as a direct result of participation in the SIMPle study, although some GPs already coded chronic conditions. GPs recognized the value of coding consultations as they perceived it as simple and good practice. Coding standardized how patient notes were recorded within the practice. The GPs also understood that if they did not code they would not receive their audit and feedback reports which contributed to fulfilling their professional competency requirements.

GPs appreciated that the SIMPle study focused on one International Classification of Primary Care code (U71) as other coding schemes use multiple codes to describe conditions and become confusing. Although all GPs expressed good intentions to code UTI consultations, remembering to code

Published by Sciedu Press every adult consultation was challenging.

"Now the only thing is my memory let me down a couple of times, the only thing I found a little bit tricky was remembering to put in the coding before going for prescribing" (GP 21.1).

The researchers had instructed the GPs to code before they prescribed for the UTI patient. This ensured that an electronic prompt was activated within their patient management software. However, this also caused some confusion as to whether the GP could go back and code a UTI patient after they had prescribed. GPs also highlighted difficulties in coding out of hours UTI consultations, nursing home visits were also perceived as difficult.

Practical demonstrations in the initial coding workshop were valued as it increased their comprehension of how and when to code U71. Two GPs interviewed were absent from the initial coding workshop and had to rely on their colleagues to train them. This highlighted a gap in the implementation of the intervention. These GPs felt it was more difficult for them to understand the rationale behind coding all UTI consultations. Other GPs mentioned minor difficulties in training new staff members who joined the practice during 
the study period. These GPs did acknowledge however, coding was very simple and the reminder text messages they received were welcomed. GPs indicated they would continue coding beyond the intervention period, some extended their consultation coding to other conditions and other GPs were undecided regarding future coding. The outcome evaluation highlighted GPs had continued to code 5 months after the intervention had concluded. ${ }^{[15]}$

Cumulative frequencies included within practice audit and feedback reports provided further evidence that practices continuously coded consultations throughout the intervention period and beyond. The follow up evaluation provided further evidence that GPs continued to code. No GP opted out of the reminder text messages related to coding or stopped coding altogether during the intervention period.

\subsection{The role of audit and feedback reports}

Although concise, the audit and feedback reports provided enough evidence to demonstrate the quality of their practices prescribing compared to other practices. GPs valued receiving practice specific data which helped them review their prescribing decisions by putting their prescribing behaviors into context. However, the amount of time spent reviewing audit and feedback reports varied from monthly to once or twice during the study period.

"I mean I would look at the SARI guidelines (Ireland's National Antimicrobial Prescribing Guideline) and I would try to stick to those but I mean being eh a participant in an actual study and getting actual positive feedback that this was, you know the SARI guideline is a guideline but getting the actual proof of the pudding, you know was very reassuring" (GP 4, female).

GPs had received little feedback on their prescribing behaviors previous to the SIMPle study which meant they had not previously reflected on their prescribing decisions. The majority of GPs admitted they had been reluctant to prescribe nitrofurantoin prior to the intervention because of perceived side effects. However, the scientific evidence provided, including the high level of resistance to trimethoprim among bacteria causing UTI in the region studied and clarification of the dosage of nitrofurantoin, had encouraged GPs to prescribe it. Once patients were satisfied GPs continued to use it.

During the interactive workshop, the researcher observed that GPs were often shocked at the level of resistance to trimethoprim, as they indicated that they had never been made aware of this level previously. In addition, when asked about prescribing broad spectrum antibiotic agents GPs were taken aback by current resistance levels but often described instances of uncertainty or complexity to justify choosing broader spectrum antibiotics for certain patients.

\subsection{SIMPle study design}

A logic model summarizing the core activities within the SIMPle study is illustrated in Figure 2 and a full description of the SIMPle study is presented elsewhere. ${ }^{[14]}$

Overall, the GPs felt the intervention was well run, professional and the instructions were clear. In most cases no additional help was requested by the practices and the monthly calls to generate reports were sufficient.

\subsection{Key messaging}

As regional trimethoprim resistance prevalence was above resistance threshold guidelines in the intervention area, ${ }^{[19]}$ nitrofurantoin was considered the only first line treatment within this context. Switching from trimethoprim to nitrofurantoin prescribing was used as an example of how improvements can be made, as well as switching from broad spectrum antibiotics (to nitrofurantoin). As such all intervention components focused on promoting the use of nitrofurantoin. Practices received a fact sheet promoting nitrofurantoin prescribing (see Figure 3) and the GPs patient management software was programmed to remind the GP to prescribe nitrofurantoin when they coded a patient U71. External to the intervention in November 2013 GPs received correspondence from University Hospital Galway which also recommended nitrofurantoin as first line treatment for a UTI.

Practices randomized to Arm B were encouraged to use a delayed prescribing strategy with their patients where appropriate. These GPs were shown a video consultation highlighting how to begin a conversation about delayed antibiotic prescribing within the time constraints of the consultation. The GPs interviewed from Arm B indicated they sometimes used delayed prescribing however, it depended on the severity of symptoms.

\section{Additional support}

The GPs were happy with the content and format of the SIMPle study as it did not interfere with the duration of the consultation. They felt that the workshops contained the "right" amount of information and the text messages they received were viewed as a friendly reminder. The electronic prompts activated upon consultation coding, received mixed reactions; some GPs found it helpful, others found it annoying as they were aware of the antibiotic prescribing guidelines. However, overall the prompt was considered a non-invasive reminder. 


\subsection{Passive participation}

In the practices with little or no change in antibiotic prescribing behaviour two distinct observations were made by the researchers. In one practice, the senior partner interviewed indicated it was he who consented to participate in the SIMPle study as he believed it would benefit the younger GPs' professional development. This partner subsequently went on sick leave without communicating his support of the study. His colleagues did not understand the rationale for the study and failed to code consultations even after receiving workshops. The GPs within this practice reported an increase in workload due to the sick leave and therefore the SIMPle study was not a priority. In another practice the partners declined to participate as they were too busy until they became aware of the benefits of the audit reports. Throughout the study researchers observed this practice's passive participation. It was difficult to schedule appointments within this practice and usually only one GP attended.

\begin{tabular}{|c|c|c|}
\hline $\begin{array}{l}\text { Funds and } \\
\text { Resources }\end{array}$ & Activities & Outcomes \\
\hline $\begin{array}{l}\text { Funding: } \\
\text { Health Research Board } \\
\text { Funding - } 3 \text { year project } \\
\text { Staffing: } \\
\text { Multidisciplinary Team: } \\
\text { Social Marketing, Health } \\
\text { Economics, } \\
\text { Microbiology, General } \\
\text { Practice } \\
\text { Principle Investigator } \\
\text { Team: } \\
\text { Epidemiology, General } \\
\text { Practice, Social } \\
\text { Marketing, } \\
\text { Microbiology, Health } \\
\text { Economics and } \\
\text { Pharmacoepidemiology } \\
\text { International Advisory } \\
\text { Board } \\
\text { Collaborations: } \\
\text { Irish Primary Care } \\
\text { Research Network } \\
\text { (IPCRN) }\end{array}$ & $\begin{array}{l}\text { Formative Research } \\
\text { Eligibility } \\
\text { - Sent urine samples to local microbiology unit } \\
\text { - Patient Management System } \\
\text { Recruitment } \\
\text { - Invitation letter, follow up phone call \& face to face } \\
\text { meeting with practice manager } \\
\text { Phase 1: Coding workshop } \\
\text { - Face to face coding workshop with practice staff } \\
\text { teaching GPs to code U71 for UTI } \\
\text { - U71 branded cupcakes } \\
\text { - Practices signed up to IPCRN } \\
\text { - GPs completed baseline questionnaire } \\
\text { Contact between workshops } \\
\text { - GPs sent reminder text messaging } \\
\text { - Practices telephoned to confirm next visit \& upload } \\
\text { first report } \\
\text { Phase 2: Interactive workshop } \\
\text { - Face to face workshop with practice staff, discussed } \\
\text { first audit report, adhering to guidelines \& benefits of } \\
\text { Nitrofurantoin } \\
\quad \text { A Arm B received additional information on } \\
\text { - A pop-up reminder is activated when U71 code is used } \\
\text { - GPs requested to send all UTI urine samples to the lab } \\
\text { \& collect patient mobile numbers (evaluation) } \\
\text { Contact between workshops } \\
\text { - Practices telephoned monthly to upload audit report } \\
\text { - Monthly reminder text messages } \\
\text { Phase } 3 \text { : Patient support } \\
\text { - iPads with 'Bug Run School days' pre-downloaded } \\
\text { - } \text { were installed in practice waiting rooms } \\
\text { - Complow up data collection } \\
\text { - }\end{array}$ & $\begin{array}{l}\text { Present scientific evidence relating to practice specific } \\
\text { data } \\
\text { - Discuss consequences of not changing } \\
\text { Reinforce good practice by comparing prescribing with } \\
\text { other participating practices } \\
\text { - Challenge prescribing norms by asking practices to } \\
\text { reconsider their rational for prescribing choices } \\
\text { - In Arm B present argument for considering UTI for } \\
\text { delayed prescribing }\end{array}$ \\
\hline
\end{tabular}

Figure 2. Logic model of the SIMPle study

\section{Discussion}

This process evaluation moved beyond evaluating size effects determined through an RCT to understanding why the effect happened. GPs engaged with the study as it was relevant to practice and the design was "simple" and easily implemented into routine care. The findings asserted the fidelity of the
SIMPle study as the GPs were satisfied with the amount of support they received and how they received it. The SIMPle study fitted into routine practice and by introducing consultation coding before the intervention began, behavioral changes were more manageable as tasks were broken down into steps. 


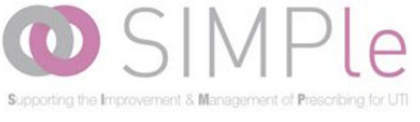

Treatment of UTI in Primary Care

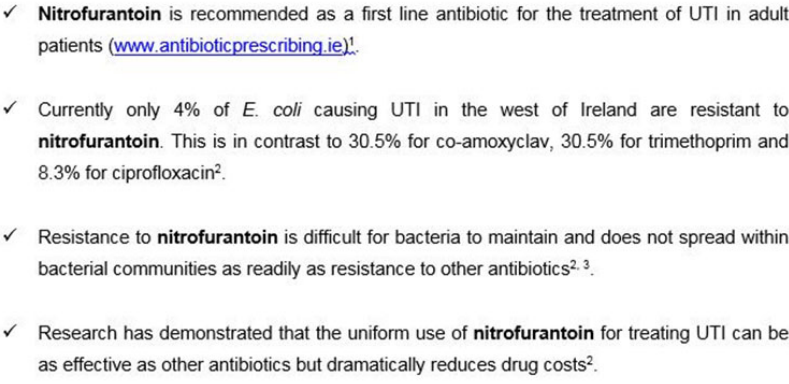

Figure 3. Nitrofurantoin factsheet distributed within intervention practices

Providing evidence of practice specific prescribing behaviors moved GPs from contemplating changes into action. Practice specific data and benchmarking antibiotic prescribing against other practices, encouraged GPs to question their normative behaviors on the basis of what they were doing now and through the provision of scientific evidence. This conscious raising exercise encouraged the GPs to re-evaluate their choices whilst providing them with alternatives. These GPs had rarely questioned why they prescribe specific antibiotics for UTI. The provision of personalized audit and feedback reports within interactive workshops opened a dialogue to discuss antibiotic decision making at practice level.

GPs were more likely to switch from trimethoprim to nitrofurantoin prescribing than from a broad spectrum antibiotic to first line. ${ }^{[15]}$ On reflection, during the interactive workshops, in the majority of cases the GPs provided an explanation for prescribing a broad spectrum antibiotic for certain patients. For most GPs these patients were perceived differently than routine UTI patients. Further research should be carried out to understand why GPs believe broad spectrum antibiotic prescribing is appropriate treatment for some UTI patients.

Unfortunately, this research provided little insight into the unintended increase in antibiotic prescribing during the intervention period as data collection took place before this outcome was known. GPs did however highlight that rational (knowledge of antibiotic prescribing guidelines) and relational (interactions with patients and empathy) factors contributed to decision making. The decision to prescribe can be complex and perhaps the benefits of prescribing nitrofurantoin communicated by the researchers (i.e. targeted treatment with low resistance) may have encouraged GPs to prescribe it more. Further research should be conducted to explain this increase.

\section{Strengths and limitations}

The planning of this process evaluation was systematic and embedded into the SIMPle study's evaluation strategy from the beginning. To ensure the sustainability of the SIMPle study in the long term, understanding why the study was successful or not was just as important as how much change took place. Both the process and outcome evaluations confirmed that GPs had improved the quality of their UTI treatment.

It was not possible to interview all GPs who participated within the SIMPle study. However, using a non-probability sampling strategy meant that a variety of GPs participated within this process evaluation. Interviews continued until theoretical saturation emerged.

\section{Conclusions}

$\mathrm{ABR}$ is as much a society problem as it is an individual one, ${ }^{[20]}$ this paper demonstrates that collective action can be successful if interventions are designed correctly. The leading systematic review in this area concluded that multifaceted interventions are more successful in changing antibiotic prescribing practices ${ }^{[11]}$ and that interactive meetings and audit reports can influence GP prescribing behaviors. These findings were supported within the SIMPle study process evaluation. It is important to note that the statistical analysis of the primary outcome highlighted the impact of the study but provided little insight as to why elements were more successful than others. The analysis of the cost effectiveness of the SIMPle study showed this was dependent on the value placed on improving antimicrobial prescribing and should be considered in terms of longer term costs. ${ }^{[21]}$ The findings from this process evaluation therefore emphasize the importance of embedding this process into RCTs. Such evaluations can assist administrators in their decision to implement behavioural interventions.

$\mathrm{ABR}$ is one consideration in the antibiotic prescribing decision making process and information on local resistance patterns provided by microbiologists is not enough to create sustainable change. ${ }^{[22]}$ Health care professionals have admitted that their prescribing may not be clinically beneficial instead are outcomes of "norms" of behaviour and the fear of the consequences of not prescribing. ${ }^{[7]}$ The SIMPle study showed that changing antibiotic prescribing is complex. Therefore it not only provided information on local resistance patterns, it also incorporated behavioral benchmarks by comparing prescribing behaviors across intervention practices. Through interactive workshops GPs were encouraged to reflect on their own behaviors and offered evidence for action. 
Sustainable behaviour change does not occur overnight and not all GPs are equally open to change. Changing antibiotic prescribing behaviour by GPs at practice level is a realistic goal which can be achieved. For behavioral interventions to be impactful within General Practice it is important that their design reflects real life practice. This involves understanding how and why behaviors are formed through rigorous formative research. Interpreting the needs of study participants using the principles of social marketing led to the design of a successful intervention which can be expanded in the future. General Practice is dynamic and often unpredictable, therefore interventions must be flexible enough to be inte- grated into routine care without much disruption yet still have impact. Overall SIMPle was successful in changing behaviour in the intended manner because the value attached to the study was relevant and made tangible through audit and feedback reports and scientific evidence. It also focused on practice level change shifting responsibility from the individual to the collective. Reflecting on prescribing behaviors through practice specific audit and feedback reports should be part of routine practice.

\section{CONFlicts OF InTEREST Disclosure}

The authors declare that they have no conflicts of interest.

\section{REFERENCES}

[1] World Health Organisation. Antimicrobial resistance: Draft global action plan on antimicrobial resistance; WHO: 2015.

[2] Anomaly J. Collective action and individual choice: Rethinking how we regulate narcotics and antibiotics. AMA J Ethics. 2013; 39: 752-756. PMid: 23427214. https://doi.org/10.1136/medeth ics-2012-101160

[3] Smith RD, Coast J. Antimicrobial resistance: A global response. Bulletin of the World Health Organization. 2002; 80: 126-133. PMid: 11953791

[4] World Health Organisation. Antimicrobial resistance: Global report on surveillance. 2014. Available from: http: //www . who. int/dr ugresistance/documents/surveillancereport/en/

[5] Murphy M, Byrne S, Bradley C. Influence of patient payment on antibiotic prescribing in irish general practice: A cohort study. $\mathrm{Br}$ J Gen Practice. 2011; 61. https : //doi .org/10.3399/bjgp11x5 93820

[6] Laxminarayan R, Duse A, Wattal C, et al. Antibiotic resistance - the need for global solutions. Lancet Infect Dis. 2013; 13: 1057-1098. https://doi.org/10.1016/S1473-3099(13)70318-9

[7] Pinder R, Sallis A, Berry D, et al. Behaviour change and antibiotic prescribing in healthcare settings: Literature review and behavioural analysis; Department of Public Health. 2015.

[8] Oakley A, Strange V, Bonell C, et al. Health services research: Process evaluation in randomised controlled trials of complex interventions. BMJ. 2006; 332: 413. PMid: 16484270. https://doi.org/ $10.1136 / \mathrm{bmj} .332 .7538 .413$

[9] Medical Research Council. Developing and evaluating complex interventions: New guidance; London. 2008.

[10] Stead M, McDermott RJ. Evaluation in social marketing. In The sage handbook of social marketing. Hastings G, Angus K, Bryant C, Eds. 2012.

[11] Arnold SR, Straus SE. Interventions to improve antibiotic prescribing practices in ambulatory care. Cochrane Database Syst Rev. 2005; 4. https://doi.org/10.1002/14651858.cd003539.pub2

[12] Grimshaw J, Zwarenstein M, Tetroe JM, et al. Looking inside the black box: A theory-based process evaluation alongside a randomised controlled trial of printed educational materials (the ontario printed educational message, opem) to improve referral and prescribing prac- tices in primary care in ontario, canada. Implementation Science. 2007; 2. https://doi .org/10.1186/1748-5908-2-38

[13] Duane S, Domegan C, Callan A, et al. Using qualitative insights to change practice - exploring the culture of antibiotic prescribing for urinary tract infections: The simple study. BMJ Open. 2016; 6 . https://doi.org/10.1136/bmjopen-2015-008894

[14] Duane S, Callan A, Galvin S, et al. Supporting the improvement and management of prescribing for urinary tract infections (simple): Protocol for a cluster randomized trial. Trials. 2013; 14: 441. PMid: 24359543. https ://doi .org/10.1186/1745-6215-14-441

[15] Vellinga A, Galvin S, Duane S, et al. Intervention to improve the quality of antimicrobial prescribing for urinary tract infection: A cluster randomized trial. CMAJ. 2015. PMid: 26573754.

[16] Moore GF, Audrey S, Barker M, et al. Process evaluation of complex interventions: Medical research council guidance. BMJ. 2015; 350 https ://doi.org/10.1136/bmj.h1258

[17] Saunders RP, Evans MH, Joshi P. Developing a process-evaluation plan for assessing health promotion program implementation: A howto guide. Health Promot Pract. 2005; 6: 134-147. PMid: 15855283. https://doi.org/10.1177/1524839904273387

[18] Prochaska JM. A transtheoretical model for assessing organizational change: A study of family service agencies' movement to timelimited therapy. FAM SOC. 2000; 81: 76-84. https ://doi.org/ 10.1606/1044-3894. 1095

[19] Vellinga A, Cormican M, Hanahoe B, et al. Antimicrobial management and appropriateness of treatment of urinary tract infection in general practice in ireland. Bmc Fam Pract. 2011; 12 https ://doi.org/10.1186/1471-2296-12-108

[20] Edgar T, Boyd SD, Palamé MJ. Sustainability for behaviour change in the fight against antibiotic resistance: A social marketing framework. J Antimicrob Chemother. 2009; 63: 230-237. PMid: 19095680. https://doi.org/10.1093/jac/dkn508

[21] Gillespie P, Callan A, O'Shea E, et al. The cost effectiveness of the simple intervention to improve antimicrobial prescribing for urinary tract infection in primary care. J Public Health (Oxf). 2016. PMid: 27679664. https : //doi.org/10.1093/pubmed/fdw102

[22] Simpson SA, Wood F, Butler C. General practitioners' perceptions of antimicrobial resistance: A qualitative study. J Antimicrob Chemother. 2007; 59: 292-296. PMid: 17110392. https : //doi.org/10.1093/jac/dkl467 